\title{
Score-Based Explanations in Data Management and Machine Learning
}

\author{
Leopoldo Bertossi ${ }^{\star}$ \\ Universidad Adolfo Ibáñez \& Data Observatory Foundation \& IMFD \\ Santiago, Chile
}

\begin{abstract}
We describe some approaches to explanations for observed outcomes in data management and machine learning. They are based on the assignment of numerical scores to predefined and potentially relevant inputs. More specifically, we consider explanations for query answers in databases, and for results from classification models. The described approaches are mostly of a causal and counterfactual nature. We argue for the need to bring domain and semantic knowledge into score computations; and suggest some ways to do this.
\end{abstract}

\section{Introduction}

In data management and machine learning one wants explanations for certain results. For example, for query results from databases, and for outcomes of classification models. Explanations, that may come in different forms, have been the subject of philosophical enquires for a long time, but, closer to home, they appear under different forms in model-based diagnosis and in causality as developed in artificial intelligence. In the last few years, explanations that are based on numerical scores assigned to elements of a model that may contribute to an outcome have become popular. These scores attempt to capture the degree of their contribution to an outcome, e.g. answering questions like these: What is the contribution of this tuple to the answer to this query? What is the contribution of this feature value of an entity to the displayed classification of the latter?

Let us consider, as an example, a financial institution that uses a learned classifier, e.g. a decision tree, to determine if clients should be granted loans or not, returning labels 0 or 1 , resp. A particular client, an entity e, applies for a loan, the classifier returns $M(\mathbf{e})=1$, i.e. the loan is rejected. The client requests an explanation. A common approach consists in giving scores to the feature values in e, to quantify their relevance in relation to the classification outcome. The higher the score of a feature value, the more explanatory is that value. For example, the fact that the client has value " 5 years" for feature Age could have the highest score.

Motivated, at least to a large extent, by the trend towards explainable AI [22], different explanation scores have been proposed in the literature. Among them, in data management, the responsibility score as found in actual causality [13, 10]

\footnotetext{
^ Email: leopoldo.bertossi@uai.cl
} 
has been used to quantify the strength of a tuple as a cause for a query result 19, 2. The Shapley-value, as found in coalition game theory, has been used for the same purpose [15]. In machine learning, in relation to results of classification models, the Shapley-value has been used to assign scores to feature values. In the form of the SHAP-score, it has become quite popular and influential [17, 18]. A responsibility-based score, RESP, was introduced in 5 to assign numbers to feature values of entities under classification. It is based on the notions of counterfactual intervention and causal responsibility.

Some scores used in machine learning appeal to the components of the mathematical model behind the classifier. There can be all kinds of explicit models, and some are easier to understand or interpret or use for this purpose. For example, the FICO-score proposed in [9, for the FICO dataset about loan requests, depends on the internal outputs and displayed coefficients of two nested logistic regression models. Decision trees [21, random forests [7, rule-based classifiers, etc., could be seen as relatively easy to understand and use for explanations on the basis of their components.

Other scores can be applied with black-box models, in that they use, in principle, only the input/output relation that represents the classifier, without having access to the internals details of the model. In this category we could find classifiers based on complex neural networks, or XGBoost [16. They are opaque enough to be treated as black-box models. The SHAP-score and the RESP-score can be applied to this category. In [5, the SHAP-score, the RESP-score and the FICO-score are compared. In general, the computation of the first two is intractable.

The SHAP-score and the RESP-score can be applied with open-box models. In this case, an interesting question is whether having access to the mathematical model may make their computation tractable, at least for some classes of classifiers.

As suggested above, scores can be assigned to tuples in databases, to measure their contribution to a query answer, or to the violation of an integrity constraint. The responsibility score has been applied for this purpose [19, 2, and is based on causality in databases [19. Also the Shapley-value has been used for this task [15.

In this article we survey some of the approaches to score-based explanations we just mentioned above, in databases and in classification in machine learning. This is not intended to be an exhaustive survey of these areas, but it is heavily influenced by our latest research. Next, we discuss the relevance of bringing domain and semantic knowledge into these score computations. We also show some first ideas and techniques on how this knowledge can be accommodated in the picture. To introduce the concepts and techniques we will use mostly examples, trying to convey the main intuitions and issues.

This paper is structured as follows. In Section 2 we concentrate on causal explanations in databases. In Section 3 , we describe the use of the Shapleyvalue to provide explanation scores in databases. In Section 3 , we describe scorebased explanations for classification results. In Section 5 we show how semantic 
knowledge can be brought into the score computations. We conclude with some final remarks in Section 6 .

\section{Explanations in Databases}

In data management we need to understand and compute why certain results are obtained or not, e.g. query answers, violations of semantic conditions, etc.; and we expect a database system to provide explanations.

\subsection{Causal responsibility}

Here, we will consider causality-based explanations [19, 20, which we will illustrate by means of an example.

Example 1. Consider the database $D$, and the Boolean conjunctive query (BCQ)

\begin{tabular}{|c|c|}
\hline \begin{tabular}{l|l|}
$R$ & $A \mid B$ \\
\end{tabular} & \begin{tabular}{l|l}
$S$ & $A$ \\
\end{tabular} \\
\hline \begin{tabular}{l|l}
$a$ & $b$
\end{tabular} & $a$ \\
\hline & $b$ \\
\hline
\end{tabular}

$$
\mathcal{Q}: \exists x \exists y(S(x) \wedge R(x, y) \wedge S(y)) .
$$

It holds: $D=\mathcal{Q}$, i.e. the query is true in $D$.

We ask about the causes for $\mathcal{Q}$ to be true: A tuple $\tau \in D$ is counterfactual cause for $\mathcal{Q}$ (being true in $D$ ) if $D \models \mathcal{Q}$ and $D \backslash\{\tau\} \not \models \mathcal{Q}$.

In this example, $S(b)$ is counterfactual cause for $\mathcal{Q}$ : If $S(b)$ is removed from $D, \mathcal{Q}$ is no longer true.

Removing a single tuple may not be enough to invalidate the query. Accordingly, a tuple $\tau \in D$ is an actual cause for $\mathcal{Q}$ if there is a contingency set $\Gamma \subseteq D$, such that $\tau$ is a counterfactual cause for $\mathcal{Q}$ in $D \backslash \Gamma$.

In this example, $R(a, b)$ is an actual cause for $\mathcal{Q}$ with contingency set $\{R(b, b)\}$ : If $R(a, b)$ is removed from $D, \mathcal{Q}$ is still true, but further removing $R(b, b)$ makes $\mathcal{Q}$ false.

Notice that every counterfactual cause is also an actual cause, with empty contingent set. Actual but non-counterfactual causes need company to invalidate a query result. Now we ask how strong are these tuples as causes? For this we appeal to the responsibility of an actual cause $\tau$ for $\mathcal{Q}[19$, defined by:

$$
\rho_{D}(\tau):=\frac{1}{|\Gamma|+1},
$$

with $|\Gamma|=$ size of a smallest contingency set for $\tau$, and 0 , otherwise.

Example 2. (ex. 1 cont.) The responsibility of $R(a, b)$ is $\frac{1}{2}=\frac{1}{1+1}$ (its several smallest contingency sets have all size 1).

$R(b, b)$ and $S(a)$ are also actual causes with responsibility $\frac{1}{2}$; and $S(b)$ is actual (counterfactual) cause with responsibility $1=\frac{1}{1+0}$. 
High responsibility tuples provide more interesting explanations. Causes in this case are tuples that come with their responsibilities as "scores". Actually, all tuples can be seen as actual causes and only the non-zero scores matter. Causality and responsibility in databases can be extended to the attribute-value level [2, 4].

There is a connection between database causality and repairs of databases w.r.t. integrity constraints (ICs) [1, and also connections to consistency-based diagnosis and abductive diagnosis. These connections have led to new complexity and algorithmic results for causality and responsibility [2, 3. Actually, the latter turns out to be intractable. In [3], causality under ICs was introduced and investigated. This allows to bring semantic and domain knowledge into causality in databases.

Model-based diagnosis is an older area of knowledge representation where explanations are main characters. In general, the diagnosis analysis is performed on a logic-based model, and certain elements of the model are identified as explanations. Causality-based explanations are somehow more recent. In this case, still a model is used, which is, in general, a more complex than a database with a query. In the case of databases, actually there is an underlying logical model, the lineage or provenance of the query [8] that we will illustrate in Section 2.2 , but it is still a relatively simple model.

The idea behind actual causality is the (potential) execution of counterfactual interventions on a structural logico-probabilistic model [13, with the purpose of answering hypothetical or counterfactual questions of the form: What would happen if we change ...? It turns out that counterfactual interventions can also be used to define different forms of score-based explanations, in the same spirit of causal responsibility in databases (c.f. Section 4.2). Score-based explanations can also be defined in the absence of a model, and without counterfactual interventions (or at least with them much less explicit).

\subsection{The causal-effect score}

Sometimes responsibility does not provide intuitive or expected results, which led to the consideration of an alternative score, the causal-effect score. We show the issues and this score by means of an example.

Example 3. Consider the database $E$ that represents the graph below, and the Boolean Datalog query $\Pi$ that is true in $E$ if there is a path from $a$ to $b$. Here, $E \cup \Pi \models$ yes.

\begin{tabular}{c|c|c|}
\hline$E$ & $X$ & $Y$ \\
\hline$t_{1}$ & $a$ & $b$ \\
$t_{2}$ & $a$ & $c$ \\
$t_{3}$ & $c$ & $b$ \\
$t_{4}$ & $a$ & $d$ \\
$t_{5}$ & $d$ & $e$ \\
$t_{6}$ & $e$ & $b$ \\
\cline { 2 - 3 }
\end{tabular}

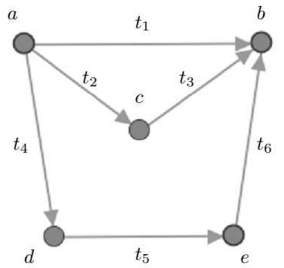

$$
\begin{aligned}
y e s & \leftarrow P(a, b) \\
P(x, y) & \leftarrow E(x, y) \\
P(x, y) & \leftarrow P(x, z), E(z, y)
\end{aligned}
$$


All tuples are actual causes since every tuple appears in a path from $a$ to $b$. Also, all the tuples have the same causal responsibility, $\frac{1}{3}$, which may be counterintuitive, considering that $t_{1}$ provides a direct path from $a$ to $b$.

In 27, the notion causal effect was introduced. It is based on three main ideas, namely, the transformation, for auxiliary purposes, of the database into a probabilistic database, interventions on the lineage of the query, and the use of expected values for the query. This is all shown in the next example.

Example 4. Consider the database $D$ below, and a BCQ.

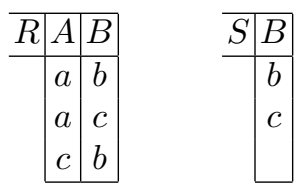

$\mathcal{Q}: \exists x \exists y(R(x, y) \wedge S(y))$, which is true in $D$.

The lineage of the query instantiated on $D$ is given by the propositional formula:

$$
\Phi_{\mathcal{Q}}(D)=\left(X_{R(a, b)} \wedge X_{S(b)}\right) \vee\left(X_{R(a, c)} \wedge X_{S(c)}\right) \vee\left(X_{R(c, b)} \wedge X_{S(b)}\right),
$$

where $X_{\tau}$ is a propositional variable that is true iff $\tau \in D$. Here, $\Phi_{\mathcal{Q}}(D)$ takes value 1 in $D$.

Now, for illustration, we want to quantify the contribution of tuple $S(b)$ to the query answer. For this purpose, we assign probabilities, uniformly and independently, to the tuples in $D$, obtaining a a probabilistic database $D^{p}$ [29]. Potential tuples outside $D$ get probability 0 .

\begin{tabular}{|c|c|c|c|}
\hline \begin{tabular}{c|c|l}
$R^{p}$ & $A$
\end{tabular} & prob & $\overline{S^{p}} B \mid$ & prob \\
\hline \begin{tabular}{l|l}
$a$ & \\
\end{tabular} & $\frac{1}{2}$ & 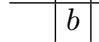 & $\frac{1}{2}$ \\
\hline \begin{tabular}{l|l}
$a$ \\
$c$
\end{tabular} & $\begin{array}{l}\frac{1}{2} \\
\frac{1}{2}\end{array}$ & $c$ & $\frac{1}{2}$ \\
\hline
\end{tabular}

The $X_{\tau}$ 's become independent, identically distributed Boolean random variables; and $\mathcal{Q}$ becomes a Boolean random variable. Accordingly, we can ask about the probability that $\mathcal{Q}$ takes the truth value 1 (or 0 ) when an intervention is performed on $D$.

Interventions are of the form $\operatorname{do}(X=x)$, meaning making $X$ take value $x$, with $x \in\{0,1\}$, in the structural model, in this case, the lineage. That is, we ask, for $\{y, x\} \subseteq\{0,1\}$, about the conditional probability $P\left(\mathcal{Q}=y \mid d o\left(X_{\tau}=x\right)\right)$, i.e. conditioned to making $X_{\tau}$ false or true.

For example, with $d o\left(X_{S(b)}=0\right)$ and $d o\left(X_{S(b)}=1\right)$, the lineage in (1) becomes, resp., and abusing the notation a bit:

$$
\begin{aligned}
& \Phi_{\mathcal{Q}}\left(D \mid d o\left(X_{S(b)}=0\right):=\left(X_{R(a, c)} \wedge X_{S(c)}\right)\right. \\
& \Phi_{\mathcal{Q}}\left(D \mid d o\left(X_{S(b)}=1\right):=X_{R(a, b)} \vee\left(X_{R(a, c)} \wedge X_{S(c)}\right) \vee X_{R(c, b)} .\right.
\end{aligned}
$$


On the basis of these lineages and $D^{p}$, when $X_{S(b)}$ is made false, the probability that the instantiated lineage becomes true in $D^{p}$ is:

$$
P\left(\mathcal{Q}=1 \mid d o\left(X_{S(b)}=0\right)\right)=P\left(X_{R(a, c)}=1\right) \times P\left(X_{S(c)}=1\right)=\frac{1}{4} .
$$

Similarly, when $X_{S(b)}$ is made true, the probability of the lineage becoming true in $D^{p}$ is:

$$
P\left(\mathcal{Q}=1 \mid d o\left(X_{S(b)}=1\right)\right)=P\left(X_{R(a, b)} \vee\left(X_{R(a, c)} \wedge X_{S(c)}\right) \vee X_{R(c, b)}=1\right)=\frac{13}{16} .
$$

The causal effect of a tuple $\tau$ is defined by:

$$
\mathcal{C E}^{D, \mathcal{Q}}(\tau):=\mathbb{E}\left(\mathcal{Q} \mid \operatorname{do}\left(X_{\tau}=1\right)\right)-\mathbb{E}\left(\mathcal{Q} \mid \operatorname{do}\left(X_{\tau}=0\right)\right) .
$$

In particular, using the probabilities computed so far:

$$
\begin{aligned}
& \mathbb{E}\left(\mathcal{Q} \mid \operatorname{do}\left(X_{S(b)}=0\right)\right)=P\left(\mathcal{Q}=1 \mid d o\left(X_{S(b)}=0\right)\right)=\frac{1}{4} \\
& \mathbb{E}\left(\mathcal{Q} \mid \operatorname{do}\left(X_{S(b)}=1\right)\right)=P\left(\mathcal{Q}=1 \mid d o\left(X_{S(b)}=1\right)\right)=\frac{13}{16} .
\end{aligned}
$$

Then, the causal effect for the tuple $S(b)$ is: $\mathcal{C E}^{D, \mathcal{Q}}(S(b))=\frac{13}{16}-\frac{1}{4}=\frac{9}{16}>0$, showing that the tuple is relevant for the query result, with a relevance score provided by the causal effect, of $\frac{9}{16}$.

Let us now retake the initial example of this section.

Example 5. (ex. 3 cont.) The Datalog query, here as a union of BCQs, has the lineage: $\Phi_{\mathcal{Q}}(D)=X_{t_{1}} \vee\left(X_{t_{2}} \wedge X_{t_{3}}\right) \vee\left(X_{t_{4}} \wedge X_{t_{5}} \wedge X_{t_{6}}\right)$. It holds:

$$
\begin{aligned}
& \mathcal{C} \mathcal{E}^{D, \mathcal{Q}}\left(t_{1}\right)=0.65625 \\
& \mathcal{C} \mathcal{E}^{D, \mathcal{Q}}\left(t_{2}\right)=\mathcal{C} \mathcal{E}^{D, \mathcal{Q}}\left(t_{3}\right)=0.21875, \\
& \mathcal{C} \mathcal{E}^{D, \mathcal{Q}}\left(t_{4}\right)=\mathcal{C} \mathcal{E}^{D, \mathcal{Q}}\left(t_{5}\right)=\mathcal{C} \mathcal{E}^{D, \mathcal{Q}}\left(t_{6}\right)=0.09375
\end{aligned}
$$

The causal effects are different for different tuples, and the scores are much more intuitive than the responsibility scores.

The definition of the causal-effect score may look rather ad hoc and arbitrary. We will revisit it in Section 3.2, where we will have yet another score for applications in databases. Actually, trying to take a new approach to measuring the contribution of a database tuple to a query answer, one can think of applying the Shapley-value, which is firmly established in game theory, and also used in several other areas.

The main idea is that several tuples together are necessary to violate an IC or produce a query result, much like players in a coalition game. Some may contribute more than others to the wealth distribution function (or simply, game function), which in this case becomes the query result, namely 1 or 0 if the query is Boolean, or a number if the query is an aggregation. The Shapley-value of a tuple can be used to assign a score to its contribution. This was done in [15], and will be retaken in Section 3.2 . But first things first. 


\section{The Shapley-Value in Databases}

\subsection{The Shapley-Value}

The Shapley value was proposed in game theory by Lloyd Shapley in 1953 [28], to quantify the contribution of a player to a coalition game where players share a wealth function 11 It has been applied in many disciplines. In particular, it has been investigated in computer science under algorithmic game theory [23, and it has been applied to many and different computational problems. The computation of the Shapley-value is, in general, intractable. In many scenarios where it is applied its computation turns out to be \#P-hard [12, 11].

In particular, the Shapley value has been used in knowledge representation, to measure the degree of inconsistency of a propositional knowledge base [14]; in data management to measure the contribution of a tuple to a query answer [15] (c.f. Section 3.2); and in machine learning to provide explanations for the outcomes of classification models on the basis of numerical scores assigned to the participating feature values [18] (c.f. Section 4.1).

Consider a set of players $D$, and a game function, $\mathcal{G}: \mathcal{P}(D) \rightarrow \mathbb{R}$, where $\mathcal{P}(D)$ the power set of $D$. The Shapley-value of player $p$ in $D$ es defined by:

$$
\operatorname{Shapley}(D, \mathcal{G}, p):=\sum_{S \subseteq D \backslash\{p\}} \frac{|S| !(|D|-|S|-1) !}{|D| !}(\mathcal{G}(S \cup\{p\})-\mathcal{G}(S)) .
$$

Notice that here, $|S| !(|D|-|S|-1)$ ! is the number of permutations of $D$ with all players in $S$ coming first, then $p$, and then all the others. That is, this quantity is the expected contribution of player $p$ under all possible additions of $p$ to a partial random sequence of players followed by a random sequence of the rests of the players. Notice the counterfactual flavor, in that there is a comparison between what happens having $p$ vs. not having it. The Shapley-value is the only function that satisfy certain natural properties in relation to games. So, it is a result of a categorical set of axioms or conditions.

\subsection{Shapley for query answering}

Back to query answering in databases, the players are tuples in the database $D$. We also have a Boolean query $\mathcal{Q}$, which becomes a game function, as follows: For $S \subseteq D$,

$$
\mathcal{Q}(S)=\left\{\begin{array}{lll}
1 & \text { if } & S \models \mathcal{Q} \\
0 & \text { if } & S \not=\mathcal{Q}
\end{array}\right.
$$

With this game elements we can define a specific Shapley-value for a database tuple $\tau$ :

$$
\operatorname{Shapley}(D, \mathcal{Q}, \tau):=\sum_{S \subseteq D \backslash\{\tau\}} \frac{|S| !(|D|-|S|-1) !}{|D| !}(\mathcal{Q}(S \cup\{\tau\})-\mathcal{Q}(S)) .
$$

\footnotetext{
1 The original paper and related ones on the Shapley value can be found in the book edited by Alvin Roth 26]. Shapley and Roth shared the Nobel Prize in Economic Sciences 2012
} 
If the query is monotone, i.e. its set of answers never shrinks when new tuples are added to the database, which is the case of conjunctive queries (CQs), among others, the difference $\mathcal{Q}(S \cup\{\tau\})-\mathcal{Q}(S)$ is always 1 or 0 , and the average in the definition of the Shapley-value returns a value between 0 and 1 . This value quantifies the contribution of tuple $\tau$ to the query result. It was introduced and investigated in [15, for BCQs and some aggregate queries defined over CQs. We report on some of the findings in the rest of this section. The analysis has been extended to queries with negated atoms in CQs 24 .

A main result obtained in [15] is in relation to the complexity of computing this Shapley score. It is the following Dichotomy Theorem: For $\mathcal{Q}$ a BCQ without self-joins, if $\mathcal{Q}$ is hierarchical, then $\operatorname{Shapley}(D, \mathcal{Q}, \tau)$ can be computed in polynomial-time (in the size of $D$ ); otherwise, the problem is $F P^{\# P}$-complete.

Here, $\mathcal{Q}$ is hierarchical if for every two existential variables $x$ and $y$, it holds: (a) $\operatorname{Atoms}(x) \subseteq \operatorname{Atoms}(y)$, or $\operatorname{Atoms}(y) \subseteq \operatorname{Atoms}(x)$, or $\operatorname{Atoms}(x) \cap$ $\operatorname{Atoms}(y)=\emptyset$. For example, $\mathcal{Q}: \exists x \exists y \exists z(R(x, y) \wedge S(x, z))$, for which $\operatorname{Atoms}(x)$ $=\{R(x, y), S(x, z)\}$, Atoms $(y)=\{R(x, y)\}, \operatorname{Atoms}(z)=\{S(x, z)\}$, is hierarchical. However, $\mathcal{Q}^{n h}: \exists x \exists y(R(x) \wedge S(x, y) \wedge T(y))$, for which Atoms $(x)=$ $\{R(x), S(x, y)\}, \operatorname{Atoms}(y)=\{S(x, y), T(y)\}$, is not hierarchical.

These are the same criteria for (in)tractability that apply to BCQs over probabilistic databases [29]. However, the same proofs do not (seem to) apply. The intractability result uses query $\mathcal{Q}^{\text {nh }}$ above, and a reduction from counting independent sets in a bipartite graph.

The dichotomy results can be extended to summation over CQs, with the same conditions and cases. This is because the Shapley-value, as an expectation, is linear. Hardness extends to aggregates max, min, and avg over non-hierarchical queries.

For the hard cases, there is an Approximation Result: For every fixed BCQ $\mathcal{Q}$ (or summation over a CQ), there is a multiplicative fully-polynomial randomized approximation scheme (FPRAS), $A$, with

$$
\left.P\left(\tau \in D \mid \frac{\operatorname{Shapley}(D, \mathcal{Q}, \tau)}{1+\epsilon} \leq A(\tau, \epsilon, \delta) \leq(1+\epsilon) \operatorname{Shapley}(D, \mathcal{Q}, \tau)\right\}\right) \geq 1-\delta .
$$

A related and popular score, in coalition games and other areas, is the Bahnzhaf Power Index, which is similar to the Shapley-value, but the order of players is ignored, by considering subsets of players rather than permutations thereof:

$$
\operatorname{Banzhaf}(D, \mathcal{Q}, \tau):=\frac{1}{2^{|D|-1}} \cdot \sum_{S \subseteq(D \backslash\{\tau\})}(\mathcal{Q}(S \cup\{\tau\})-\mathcal{Q}(S)) .
$$

The Bahnzhaf-index is also difficult to compute; provably \#P-hard in general. The results in [15] carry over to this index when applied to query answering in databases.

In [15] it was proved that the causal-effect score of Section 2.2 coincides with the Banzhaf-index, which gives to the former a more fundamental or historical justification. 


\section{Score-Based Explanations for Classification}

Let us consider a classifier, $\mathcal{C}$, that receives a representation of a entity, e, as a record of feature values, and outputs a label, $L(\mathbf{e})$, corresponding to the possible decision alternatives. We could see $\mathcal{C}$ as a black-box, in the sense that only by direct interaction with it, we have access to its input/output relation. We may not have access to the mathematical classification model inside $\mathcal{C}$.

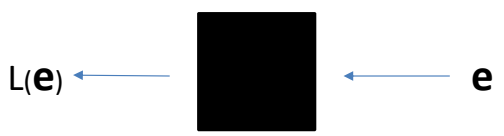

To simplify the presentation we will assume that the entities and the classifier are binary, that is, in the representation $\mathbf{e}=\left\langle x_{1}, \ldots, x_{n}\right\rangle$ of an entity, the feature values are binary ( 0 or 1 ), corresponding to propositional features (false or true, resp.). The label is always 0 or 1 . For example, we could have a client of a financial institution requesting a loan, but the classifier, on the basis of his/her feature values, assigns the label 1 , for rejection. An explanation is requested by the client. Of course, the same situation may occur if we have an explicit classification model, e.g. a classification tree or a logistic regression model, in which cases, we might be in a better position to given an explanation, because we can inspect the internals of the model 25. However, we will put ourselves in the "worst scenario" in which we do not have access to the internal model.

An approach to explanations that has become popular, specially in the absence of the model, assigns numerical scores, trying to answer the question about which of the feature values $x_{i}$ of $\mathbf{e}$ contribute the most to the received label.

Score-based methodologies are sometimes based on counterfactual interventions: What would happen with the label if we change this value, leaving the others fixed? Or the other way around: What if we leave this value fixed, and change the others? The resulting labels can be aggregated, leading to a score for the feature value under inspection.

In the next two sections we briefly introduce two scores. Both can be applied with open-box or black-box models.

\subsection{The SHAP-score}

We will consider until further announcement the uniform probability space. Actually, since we consider only binary feature values taking values 0 or 1 , this is the uniform distribution on $E=\{0,1\}^{n}$, assigning probability $P^{u}(\mathbf{e})=\frac{1}{2^{n}}$ to $\mathbf{e} \in E$. One could consider appealing to other, different distributions.

In the context of classification, the Shapley-value has taken the form of the SHAP-score [17, which we briefly introduce. Given the binary classifier, $\mathcal{C}$, on binary entities, it becomes crucial to identify a suitable game function. In this case, it will be expressed in terms of expected values (not unlike the causaleffect score), which requires an underlying probability space on the population of entities. For the latter we use, as just said, the uniform distribution over $\{0,1\}^{n}$. 
Given a set of features $\mathcal{F}=\left\{F_{1}, \ldots, F_{n}\right\}$, and an entity e whose label is to be explained, the set of players $D$ in the game is $\mathcal{F}(\mathbf{e}):=\{F(\mathbf{e}) \mid F \in \mathcal{F}\}$, i.e. the set of feature values of $\mathbf{e}$. Equivalently, if $\mathbf{e}=\left\langle x_{1}, \ldots, x_{n}\right\rangle$, then $x_{i}=F_{i}(\mathbf{e})$. We assume these values have implicit feature identifiers, so that duplicates do not collapse, i.e. $|\mathcal{F}(\mathbf{e})|=n$. The game function is defined as follows. For $S \subseteq \mathcal{F}(\mathbf{e})$,

$$
\mathcal{G}_{\mathbf{e}}(S):=\mathbb{E}\left(L\left(\mathbf{e}^{\prime}\right) \mid \mathbf{e}_{S}^{\prime}=\mathbf{e}_{S}\right),
$$

where $\mathbf{e}_{S}$ : is the projection of $\mathbf{e}$ on $S$. That is, the expected value of the label for entities $\mathbf{e}^{\prime}$ when their feature values are fixed and equal to those in in $S$ for e. Other than that, the feature values of $\mathbf{e}^{\prime}$ may independently vary over $\{0,1\}$.

Now, one can instantiate the general expression for the Shapley-value in (2), using this game function, as Shapley $\left(\mathcal{F}(\mathbf{e}), \mathcal{G}_{\mathbf{e}}, F(\mathbf{e})\right)$, obtaining, for a particular feature value $F(\mathbf{e})$ :

$$
\begin{aligned}
& \operatorname{SHAP}\left(\mathcal{F}(\mathbf{e}), \mathcal{G}_{\mathbf{e}}, F(\mathbf{e})\right):=\sum_{S \subseteq \mathcal{F}(\mathbf{e}) \backslash\{F(\mathbf{e})\}} \frac{|S| !(n-|S|-1) !}{n !} \times \\
& \left(\mathbb{E}\left(L\left(\mathbf{e}^{\prime} \mid \mathbf{e}_{S \cup\{F(\mathbf{e})\}}^{\prime}=\mathbf{e}_{S \cup\{F(\mathbf{e})\}}\right)-\mathbb{E}\left(L\left(\mathbf{e}^{\prime}\right) \mid \mathbf{e}_{S}^{\prime}=\mathbf{e}_{S}\right)\right) .\right.
\end{aligned}
$$

Here, the label $L$ acts as a Bernoulli random variable that takes values through the classifier. We can see that the SHAP-score is a weighted average of differences of expected values of the labels [17.

\subsection{The RESP-score}

In the same setting of Section 4.1, let us consider the following score introduced in [5. For $F \in \mathcal{F}$, and an entity e for which we have obtained label 1, the "negative" outcome one would like to see explained:

$$
\operatorname{COUNTER}(\mathbf{e}, F):=L(\mathbf{e})-\mathbb{E}\left(L\left(\mathbf{e}^{\prime}\right) \mid \mathbf{e}_{\mathcal{F} \backslash\{F\}}^{\prime}=\mathbf{e}_{\mathcal{F} \backslash\{F\}}\right) .
$$

This score measures the expected difference between the label for e and those for entities that coincide in feature values everywhere with e but on feature $F$. Notice the essential counterfactual nature of this score, which is reflected in all the possible hypothetical changes of features values in e.

The COUNTER-score can be applied in same scenarios as SHAP, it is easier to compute, and gives reasonable and intuitive results, and also behaves well in experimental comparisons with other scores [5. As with the SHAP-score, one could consider different underlying probability distributions (c.f. [5] for a discussion). Again, so as for SHAP, there is no need to access the internals of the classification model.

One problem with COUNTER is that changing a single value, no matter how, may not switch the original label, in which case no explanations are obtained. In order to address this problem, we can bring in contingency sets of feature values, which leads to the RESP-score introduced in [5]. We just give the idea and a simplified version of it by means of an example. 
Example 6. In the picture below, the black box is the classifier. Entities have three feature values. The table on the right-hand side shows all the possible entities with their labels. We want to explain the label 1 obtained by entity $\mathbf{e}_{1}$.

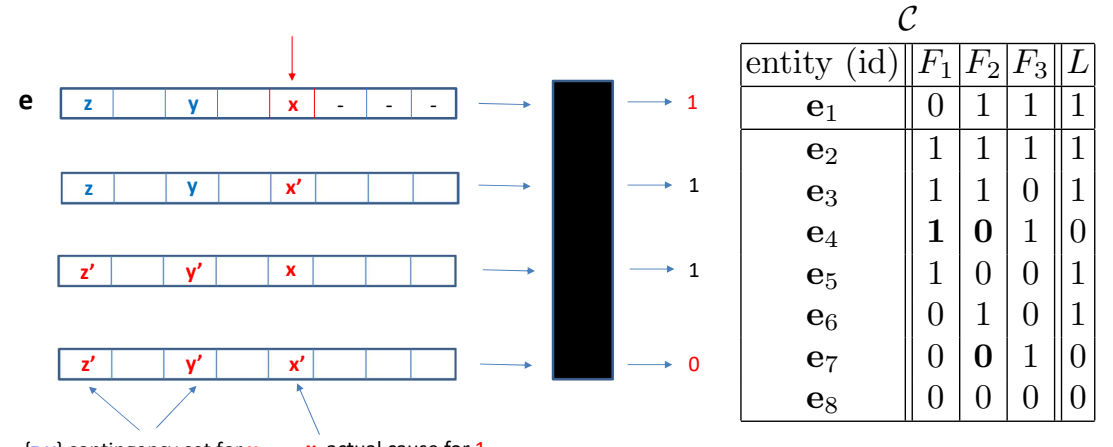

Through counterfactual interventions we change feature values in $\mathbf{e}_{1}$, trying to change the label to 0 . This process is described in the figure above, on the left-hand side, where we are attempting to quantify the contribution of value $\mathbf{x}=F\left(\mathbf{e}_{1}\right)$. Let us assume that by changing $\mathbf{x}$ into any $\mathbf{x}^{\prime}$, we keep obtaining label 1. So, we leave $\mathbf{x}$ as it is, and consider changing other original values, $\mathbf{y}$ and $\mathbf{z}$, into $\mathbf{y}^{\prime}$ and $\mathbf{z}^{\prime}$, still getting 1 . However, if we now, in addition, change $\mathbf{x}$ into $\mathbf{x}^{\prime}$, we get label 0. Then, in the spirit of actual causality, as seen in Section 2.1, we can say that the feature value $\mathbf{x}$ is an actual cause for the original label 1 , with $\mathbf{y}$ and $\mathbf{z}$ forming a contingency set for $\mathbf{x}$; in this case, of size 2 .

On this basis, we can define 6]: (a) $\mathbf{x}$ is a counterfactual explanation for $L(\mathbf{e})=1$ if $L\left(\mathbf{e} \frac{\mathbf{x}}{\mathbf{x}^{\prime}}\right)=0$, for some $\mathbf{x}^{\prime} \in \operatorname{Dom}(F)$ (the domain of feature $F$ ). (b) $\mathbf{x}$ is an actual explanation for $L(\mathbf{e})=1$ if there is a set of values $\mathbf{Y}$ in $\mathbf{e}$, with $\mathbf{x} \notin \mathbf{Y}$, and new values $\mathbf{Y}^{\prime} \cup\left\{\mathbf{x}^{\prime}\right\}$, such that $L\left(\mathbf{e} \frac{\mathbf{Y}}{\mathbf{Y}^{\prime}}\right)=1$ and $L\left(\mathbf{e} \frac{\mathbf{x Y}}{\mathbf{x}^{\prime} \mathbf{Y}^{\prime}}\right)=0$. Here, as usual, $\frac{\mathbf{x}}{\mathbf{x}^{\prime}}$, denotes the replacement of value $\mathbf{x}$ by $\mathbf{x}^{\prime}$, and so on.

Contingency sets may come in sizes from 0 to $n-1$ for feature values in records of length $n$. Accordingly, we can define for the actual cause $\mathbf{x}$ : If $\mathbf{Y}$ is a minimum size contingency set for $\mathbf{x}, \operatorname{RESP}(\mathbf{x}):=\frac{1}{1+|\mathbf{Y}|}$; and as 0 when $\mathbf{x}$ is not an actual cause. This score can be formulated in terms of expected values, generalizing expression (3) through the introduction of contingency sets [5].

Coming back to the entities in the figure above, due to $\mathbf{e}_{7}, F_{2}\left(\mathbf{e}_{1}\right)$ is counterfactual explanation; with $\operatorname{RESP}\left(F_{2}\left(\mathbf{e}_{1}\right)\right)=1$. Due to $\mathbf{e}_{4}, F_{1}\left(\mathbf{e}_{1}\right)$ is actual explanation; with $\left\{F_{2}\left(\mathbf{e}_{1}\right)\right\}$ as contingency set, and $\operatorname{RESP}\left(F_{1}\left(\mathbf{e}_{1}\right)\right)=\frac{1}{2}$.

\section{Bringing-In Domain Knowledge}

The uniform space gives equal probability to each entity in the underlying population. One can argue that this is not realistic, in that certain combinations of feature values may be more likely than others; or that certain correlations 
among them exist. One can consider assigning or modifying probabilities in the hope of capturing correlations and logical relationships between feature values.

\subsection{Empirical Distributions}

An alternative consists in using an empirical distribution as a proxy. In this case we have a sample $S \subseteq E$ (we could have repetitions, but we do not consider this case here). The probability of $\mathbf{e} \in E$, is given by:

$$
P_{S}(\mathbf{e}):=\left\{\begin{array}{cl}
\frac{1}{|S|} & \text { if } \mathbf{e} \in S \\
0 & \text { if } \mathbf{e} \notin S
\end{array}\right.
$$

The empirical distribution was used in 5] to compute the SHAP-score. More precisely, the entities in $S$ come with labels obtained via the classifier $\mathcal{C}$; and the score is computed with expectations directly with the entities in $S$, with their labels. The empirical distribution may be better at capturing correlations.

\subsection{Imposing Constraints}

One can introduce constraints that prohibit certain combinations of values, in the spirit of denial constraints in databases, but in this case admitting positive and negative atoms. For example, we may not want the combination of "The age is not greater than 20" and "Gets an account overdraft above \$50M" to hold simultaneously.

These constraints, which are satisfied or violated by a single entity at a time, are of the form:

$$
\chi: \neg\left(\bigwedge_{i \in S} F_{i} \wedge \bigwedge_{j \in S^{\prime}} \bar{F}_{j}\right),
$$

where $S \cup S^{\prime} \subseteq F, S \cap S^{\prime}=\emptyset$, and $F_{i}, \bar{F}_{j}$ mean that features $F_{i}, F_{j}$ take values 1 and 0 , resp. In the example, it would be of the form $\neg(\overline{A g e} \wedge$ OverDr50M $)$. The events, i.e. subsets of $E$, associated to the violation of $\chi$ should get zero probability.

A way to accommodate a constraint, $\chi$, consists in defining an event associated to it:

$$
A(\chi)=\{\mathbf{e} \in E \mid \mathbf{e} \models \chi\},
$$

where $\mathbf{e}=\chi$ has the obvious meaning of satisfaction of $\chi$ by entity $\mathbf{e}$.

Given the uniform probability space $\left\langle E, P^{u}\right\rangle$, we can redefine the probability in order to enforce $\chi$. For $A \subseteq E$,

$$
P_{\chi}^{u}(A):=P^{u}(A \mid A(\chi))=\frac{P^{u}(A \cap A(\chi))}{P^{u}(A(\chi))} .
$$

Since $\chi$ is logically consistent (it is satisfied by some entities in $E$ ), the conditional distribution is well-defined. Notice that the probability of $\chi$ 's violation set, i.e. of $E \backslash A(\chi)$, is now:

$$
P_{\chi}^{u}(E \backslash A(\chi))=\frac{P^{u}(\emptyset)}{P^{u}(A(\chi))}=0 .
$$


This definition can be extended to finite sets, $\Theta$, of constraints, as long as it is consistent (i.e. satisfiable in $E$ ), by using $\wedge \Theta$, the conjunction of the constraints in $\Theta: P_{\Theta}^{u}(A):=P_{\wedge \Theta}^{u}(A)$.

Of course, one could go beyond constraints of the form (5), applying the same ideas, and consider any propositional formula that is intended to be evaluated on a single entity at a time, as opposed to considering combinations of feature values for different entities.

The resulting modified distributions that accommodate constraints could be used in the computation of any of the scores expressed in terms of expected values (or in probabilistic terms, in general).

\section{Final Remarks}

Explainable AI (XAI) is an effervescent area of research. Its relevance can only grow considering that legislation around explainability, transparency and fairness of AI/ML systems is being produced and enforced. There are different approaches and methodologies in relation to explanations, and causality, counterfactuals and scores have a relevant role to play.

Much research is still needed on the use of contextual, semantic and domain knowledge. Some approaches may be more appropriate, e.g. declarative ones [6].

Still fundamental research is needed on what is a good explanation, and in particular, on what are the desired properties of an explanation score. After all, the original, general Shapley-value emerged from a list of desiderata in relation to coalition games. Although the Shapley value is being used in XAI, in particular in its SHAP incarnation, there could be a different and specific set of desired properties of explanation scores that could lead to a still undiscovered explanation-score.

Acknowledgments: L. Bertossi is a member of the Academic Network of RelationalAI Inc., where his interest in explanations in ML started.

\section{References}

[1] Bertossi. L. Database Repairing and Consistent Query Answering. Synthesis Lectures in Data Management. Morgan \& Claypool, 2011.

[2] Bertossi, L. and Salimi, B. From causes for database queries to repairs and modelbased diagnosis and back. Theory of Computing Systems, 2017, 61(1):191-232.

[3] Bertossi, L. and Salimi, B. Causes for query answers from databases: datalog abduction, view-updates, and integrity constraints. Int. J. Approximate Reasoning, 2017, 90:226-252.

[4] Bertossi, L. Characterizing and computing causes for query answers in databases from database repairs and repair programs. Proc. FoIKs, 2018, Springer LNCS 10833, pp. 55-76. Extended version posted as arXiv:1712.01001, 2020.

[5] Bertossi, L., Li, J., Schleich, M., Suciu, D. and Vagena, Z. Causality-based explanation of classification outcomes. In Proceedings of the Fourth Workshop on Data Management for End-To-End Machine Learning, DEEM@SIGMOD 2020, pages 6:1-6:10, 2020. 
[6] Bertossi, L. An ASP-based approach to counterfactual explanations for classification. To appear in Proc. RuleML-RR'20. arXiv:2004.13237, 2020.

[7] L. Breiman, J. Friedman, C. J. Stone, and R. A. Olshen. Classification and regression trees. CRC press, 1984.

[8] Buneman, P., Khanna, S. and Tan, W. C. Why and where: a characterization of data provenance. Proc. ICDT, 2001, pp. 316-330.

[9] Chen, C., Lin, K., Rudin, C., Shaposhnik, Y., Wang, S. and Wang, T. An interpretable model with globally consistent explanations for credit risk. CoRR, abs/1811.12615, 2018.

[10] Chockler, H. and Halpern, J. Responsibility and blame: A structural-model approach. J. Artif. Intell. Res., 22:93-115, 2004.

[11] Deng, X. and Papadimitriou, C. On the complexity of cooperative solution concepts. Math. Oper. Res., 19(2):257-266, 1994.

[12] Faigle, U. and Kern, W. The shapley value for cooperative games under precedence constraints. International Journal of Game Theory, 21:249-266, 1992.

[13] Halpern, J. and Pearl, J. Causes and explanations: A structural-model approach. part i: Causes. The British journal for the philosophy of science, 56(4):843-887, 2005.

[14] Hunter, A. and Konieczny, S. On the measure of conflicts: Shapley inconsistency values. Artif. Intell., 174(14):1007-1026, 2010.

[15] Livshits, E., Bertossi, L., Kimelfeld, B. and Sebag, M. The Shapley value of tuples in query answering. In 23rd International Conference on Database Theory, ICDT 2020, March 30-April 2, 2020, Copenhagen, Denmark, volume 155, pages 20:1-20:19, 2020.

[16] Lucic, A., Haned, H. and de Rijke, M. Explaining predictions from tree-based boosting ensembles. CoRR, abs/1907.02582, 2019.

[17] Lundberg, S., Erion, G., Chen, H., DeGrave, A., Prutkin, J., Nair, B., Katz, R., Himmelfarb, J., Bansal, N. and Lee, S.-I. From local explanations to global understanding with explainable ai for trees. Nature machine intelligence, 2(1):25225839, 2020.

[18] Lundberg, S. and Lee, S. A unified approach to interpreting model predictions. In Advances in Neural Information Processing Systems 30: Annual Conference on Neural Information Processing Systems 2017, 4-9 December 2017, Long Beach, $C A, U S A$, pages 4765-4774, 2017.

[19] Meliou, A., Gatterbauer, W., Moore, K. F. and Suciu, D. The complexity of causality and responsibility for query answers and non-answers. Proc. VLDB, 2010, pp. 34-41.

[20] Meliou, A., Gatterbauer, W., Halpern, J.Y., Koch, C., Moore, K. F. and Suciu, D. Causality in databases. IEEE Data Eng. Bull., 2010, 33(3):59-67.

[21] Mitchell, T. M. Machine Learning. McGraw Hill series in computer science. McGraw-Hill, 1997.

[22] Molnar, C. Interpretable Machine Learning: A Guide for Making Black Box Models Explainable. https://christophm.github.io/interpretable-ml-book, 2020.

[23] Nisan, N., Roughgarden, T., Tardos, E. and Vazirani, V. V. (eds.) Algorithmic Game Theory. Cambridge University Press, 2007.

[24] Reshef, A., Kimelfeld, B. and Livshits, E. The impact of negation on the complexity of the shapley value in conjunctive queries. Proc. PODS 2020, pp. 285-297.

[25] Rudin, C. Stop explaining black box machine learning models for high stakes decisions and use interpretable models instead. Nature Machine Intelligence, 2019, 1:206-215. Also arXiv:1811.10154,2018. 
[26] Roth, A. E. (ed.) The Shapley Value: Essays in Honor of Lloyd S. Shapley. Cambridge University Press, 1988.

[27] Salimi, B., Bertossi, L., Suciu, D. and Van den Broeck, G. Quantifying causal effects on query answering in databases. Proc. 8th USENIX Workshop on the Theory and Practice of Provenance (TaPP), 2016.

[28] Shapley, L. S. A value for n-person games. Contributions to the Theory of Games, 2(28):307-317, 1953.

[29] Suciu, D., Olteanu, D., Re, C. and Koch, C. Probabilistic Databases. Synthesis Lectures on Data Management, Morgan \& Claypool, 2011. 